\title{
RENORMALIZATION GROUP CALCULATION FOR CRITICAL POINTS OF HIGHER ORDER WITH GENERAL PROPAGATOR ${ }^{\text {h }}$
}

\author{
J.F. NICOLL, G.F. TUTHILL, T.S. CHANG and H.E. STANLEY \\ Physics Department, Massachusetts Institute of Technology, Cambridge, Massachusetts 02139, USA
}

Received 9 June 1976

\begin{abstract}
We give first order perturbation results for the critical point exponents at order $O$ critical points with anisotropic propagators. The exponent $\eta$ is calculated to second order for isotropic propagators, and all $O ; 1 / n$ expansion results are given for $O=2$.
\end{abstract}

Recently, Hornreich, Luban and Shtrikman have used renormalization group techniques to discuss the onset of helical order in magnetic systems $[1,2]$. In particular, the existence of new types of critical behavior has been postulated for the "Lifshitz" point where the transition from a uniformly ordered to a helically ordered state occurs. At such a point, the propagator differs from the usual Wilson form, $G^{-1}$ $=k^{2}+r$. Refs. [1-2] consider propagators of the form $G^{-1}=k_{1}^{2}+k_{2}^{4}+r$ where $k_{i}$ is a $d_{i}$-dimensional wave vector, and $d_{1}+d_{2}=d$, the dimension of the lattice. form

Here we consider critical propagators $(r=0)$ of the

$G^{-1}=\sum_{i=1}^{J}\left|k_{i}\right|^{\sigma_{i}}$,

where each $k_{i}$ is a $d_{i}$-dimensional vector, so that $\sum_{i=1}^{J} d_{i}=d$. The $\sigma_{i}$ are termed "propagator exponents". Renormalization group techniques are applied to systems described by (1) in a manner parallel to earlier work [3-4].

For an isotropic $n$-vector Wilson model at an $O$ th order [5] critical point the borderline dimension $d_{\mathrm{b}}$ (above which mean field behavior holds) is determined by

$\sum_{i=1}^{J} d_{i} / \sigma_{i}=O /(O-1)$.

Catastrophic infrared divergences set in at dimensions below $d_{\min }$ at which

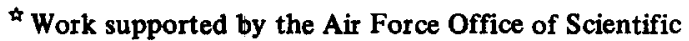
Research and the National Science Foundation.
}

$$
\sum_{i=1}^{J} d_{i} / \sigma_{i}=1 \text {. }
$$

For $\sigma_{i}=2$ these conditions reduce to those given previously $[5,6]$. In some cases (such as isotropic propagators $[1-3]) d_{\min }$ may be larger than three. A more interesting physical case is obtained if only one component of $k$ enters $G^{-1}$ as $k^{2 L}$ and the remaining components have $k^{2}$ dependence. Eqs. (2)-(3) then give $d_{\mathrm{b}}=(3 O-1) /(O-1)-1 / L$ and $d_{\min }=3-1 / L$. Thus, we have $d_{\mathrm{b}} \geqslant 3>d_{\min }$ for all $O \leqslant 2 L+1$.

For anisotropic systems, the critical point exponents $\left\{\eta_{i}\right\}$ are defined by examining the behavior of the critical two-point function for a wave-vector lying entirely in one of the $d_{i}$-dimensional subspaces:

$\Gamma_{2}\left(k_{i}\right) \propto\left|k_{i}\right|^{\sigma_{i}-\eta_{i}}$.

There will also be different values of the correlation length exponent $\nu_{i}$ in each of the subspaces. The following relationships between the exponents hold generally

$2-\alpha=\sum_{i=1}^{J} d_{i} \nu_{i} ; \quad \gamma=\left(\sigma_{i}-\eta_{i}\right) \nu_{i} ;$

$\delta=\frac{\sum_{i=1}^{J} d\left(\sigma_{i}-\eta_{j}\right)+1}{\sum_{j=1}^{J} d_{j} /\left(\sigma_{j}-\eta_{j}\right)-1}$.

Denoting the largest of the propagator exponents as $\sigma_{>}$, we define the unperturbed or Gaussian eigenvalues $\lambda_{p}$ (corresponding to $s^{2 p}, \mathrm{cf}[3]$ )

$\lambda_{p} \equiv\left[\sum_{i=1}^{J} d_{i} \sigma_{>} / \sigma_{i}\right](1-p)+p \sigma_{>}$. 
The expansion parameter for $d<d_{\mathrm{b}}$ is $\epsilon_{O} \equiv \lambda_{O}$. The corrected eigenvalues $\lambda_{p}^{\prime}$ for this general anisotropic case are found to be

$\lambda_{p}^{\prime}=\lambda_{p}-2 \epsilon_{O}\langle O, p ; p\rangle_{n} /\langle O, O ; O\rangle_{n}$,

where $[3,7]$

$\langle O, p ; p\rangle_{n} \equiv \sum_{j=0}^{[O / 2]}\left(\begin{array}{l}p \\ j\end{array}\right)\left(\begin{array}{c}p+\frac{1}{2} n-1 \\ j\end{array}\right)\left(\begin{array}{c}2 p-2 j \\ O-2 j\end{array}\right)$.

The calculation of the $\left\{\eta_{i}\right\}$ is more difficult except for the somewhat unphysical isotropic case (if $G^{-1}$ $=k^{\sigma}$, then $d_{\min }=\sigma$ ). We find that for $\sigma \neq 2 L$, there is no shift in the propagator exponent, i.e. $\eta=0$ to $\mathrm{O}\left(\epsilon_{O}^{2}\right)$. For $\sigma=2 L$ (the generalized Lifshitz point [3]), we find at an $O$ th order critical point [8]

$\eta_{O}=\frac{4(-1)^{L+1} \epsilon_{O}^{2}\left(O \Gamma^{2}\left(\frac{1}{2} d_{\mathrm{b}}\right)\right) C_{n}}{L\left(\begin{array}{c}2 O \\ O\end{array}\right)^{3} \Gamma\left(\frac{1}{2} d_{\mathrm{b}}-L\right) \Gamma\left(\frac{1}{2} d_{\mathrm{b}}+L\right)}$,

with

$C_{n} \equiv\left[\frac{\langle, O ; O\rangle_{n}}{\langle O, O ; O\rangle_{n=1}^{-}}\right]^{2} \prod_{j=1}^{O-1} \frac{2 j+n}{2 j+1}$.

Here, $d_{\mathrm{b}}=2 L O /(O-1)$ and $\epsilon_{O}=\left(d_{\mathrm{b}}-d\right)(O-1)$.

For the ordinary critical point $(O=2)$, we write simply $\epsilon_{2}=\epsilon=4 L-d$. Eq. (8) reduces to

$\eta=\frac{(-1)^{L+1} \Gamma^{2}(2 L)(n+2) \epsilon^{2}}{-L+(L)+(3 L)(n+8)^{2}}+O\left(\epsilon^{3}\right)$.
For this case we have also calculated the leading term in the $1 / n$ expansion for all $L$ (ref. [2] considered the $L=2$ case). The result is

$$
\begin{aligned}
& \eta_{2}(2 L) \frac{(-1)^{L+1}}{L} \frac{\epsilon \sin \pi \epsilon / 2}{\pi / 2} \frac{\Gamma(d-2 L) \Gamma(2 L)}{\Gamma\left(\frac{1}{2} d+L\right) \Gamma\left(\frac{1}{2} d-L\right)} \frac{1}{n} \\
& \quad+\mathrm{O}\left(1 / n^{2}\right) .
\end{aligned}
$$

In eq. (10), $\epsilon$ is not restricted to be small. Agreement between (9) and (10) is obtained for $\epsilon \ll 1$ and $n \gg 1$. Work is in progress on the general anisotropic case.

\section{References}

[1] R.M. Hornreich, M. Lubon and S. Shtrikman, Phys. Rev. Lett. 35 (1975) 1678.

[2] R.M. Hornreich, M. Luban and S. Shtrikman, Phys. Lett. 55A (1975) 269.

[3] J.F. Nicoll, T.S. Chang and H.E. Stanley, Phys. Rev. A 13 (1976) 1251.

[4] K.G. Wilson and J. Kogut, Phys. Rep. 12 (1974) 73 and references contained therein.

[5] T.S. Chang, G.F. Tuthill and H.E. Stanley, Phys. Rev. B9 (1974) 4882.

[6] J.F. Nicoll, T.S. Chang and H.E. Stanley, Phys. Rev. Lett. 33 (1974) 427.

[7] F.J. Wegner, Phys. Lett. 54A (1975) 1.

[8] The calculations are a simple extension of the method described in G.F. Tuthill, J.F. Nicoll and H.E. Stanley, Phys. Rev. B11 (1975) 4579. 